\title{
OBSERVATIONS SUR LE PARASITISME D'IPS SEXDENTATUS (INSECTA : SCOLYTIDAE) PAR PARASITORHABDITIS IPSOPHILA (NEMATODA : RHABDITIDAE)
}

\author{
F. LIEUTIER* (1) \\ Collaboration technique : Michèle Jastrabsky et Paulette Bonnafe
}

RÉSUMÉ. L'étude des pourcentages de parasitisme et de l'intensité des contaminations d'Ips sexdentatus par $P$. ipsophila, ainsi que l'examen de la vermoulure des galeries du Scolyte permettent de préciser certains caractères de la biologie du nématode. Les larves d'I. sexdentatus peuvent être contaminées, mais pas les nymphes. Les adultes sont infestés dès les premiers stades de leur maturation, les contaminations se poursuivant pendant toute la maturation de préessaimage. Après l'essaimage et leur installation sur un nouvel arbre, les insectes se décontaminent rapidement, mais des recontaminations semblent possibles en fin de ponte. Dans les insectes, les larves de $P$. ipsophila se localisent d'abord dans le mésentéron, puis gagnent l'intestin postérieur. Il ne semble pas y avoir d'évolution du parasite dans son hôte. Tous les stades de développement du nématode sont observés dans les galeries du Scolyte pendant toute la période de présence de celui-ci. Aucune relation apparente n'existe entre le parasitisme du tube digestif par P. ipsophila et celui de la cavité générale ou du tissu adipeux par les Parasitaphelenchus ou Contortylenchus diplogaster. L'action de $P$. ipsophila sur les populations d' $I$. sexdentatus est très limitée. Un léger retard dans l'essaimage et dans l'initiation de la ponte a pu être observé, ainsi qu'une très faible diminution de la densité des encoches de ponte et des œufs, mais les caractères de la galerie de ponte (longueur totale, longueur avant la première encoche) ne paraissent pas modifiés. Aucune, mortalité n'a pu être décelée.

\section{Observations on the parasitism of Ips sexdentatus (Insecta : Scolytidae) by Parasitorhabditis ipsophila (Nematoda : Rhabditidae)}

SUMMARY. The study of parasitism percentages and contaminations intensity in Ips sexdentatus parasitized by $P$. ipsophila as well as the examination of the frass in the galleries of the bark beetle gave better insight into certain features of nematode biology. Larvae of $I$. sexdentatus could be infected, whereas pupae could not. Adults were contaminated from initial stages of maturation and throughout preswarming maturation. Following swarming and installation on a new tree, insects were rapidly decontaminated, but recontamination could occur by the end of oviposition. P. ipsophila larvae were found in the mesenteron before they penetrated into the hind gut. Seemingly, the parasite underwent no evolution within its host. All developmental stages of the nematode could be observed in the galleries of the bark beetle as long as the latter

* Institut National Agronomique, Laboratoire de Zoologie, 16 rue Claude-Bernard, 575005 Paris.

(1) Adresse actuelle : INRA, Station de Zoologie et de Biocénotique forestière, Centre de Recherche Forestière d'Orléans, Ardon, F 45160 Olivet.

Accepté le 23 décembre 1983 . 
was present. No apparent relation exists between parasitism of the digestive tract by $P$. ipsophila and parasitism of the body cavity or fat body by Parasitaphelenchus or Contortylenchus diplogaster. P. ipsophila exerts very limited effects on I. sexdentatus populations. A slight delay in swarming and initiation of oviposition, and a very low decrease in density of notches of oviposition and of eggs was observed, but the features of the gallery of oviposition (total length, length before the first notch) showed no alteration. No mortality was detected.

Parasitorhabditis ipsophila Lieutier et Laumond, 1978, se rencontre très fréquemment dans les galeries et dans le tube digestif d'Ips sexdentatus Boern. (Lieutier et Laumond, 1978 ; Lieutier, 1979a.) Dans le cadre d'une étude des relations hôteparasite entre ce Scolytide et ses nématodes associés, il nous paraît utile d'apporter quelques informations sur la biologie de $P$. ipsophila et de déterminer quelle action ce dernier peut exercer sur les populations hôtes.

Les nématodes du tube digestif des Scolytides appartiennent tous au gente Parasitorhabditis et, selon Lazarevskaja (1965a) et Slobodyanyuc (1974), représentent un groupe écologique distinct des Parasitorhabditis des tubes de Malpighi ou de la cavité générale. Dans le tube digestif de leur hôte, ils se rencontrent sous forme de larves de deuxième stade et ne subiraient aucun développement (Fuchs, 1937; Slobodyanyuc, 1974), tout le cycle se déroulant dans les galeries du Scolyte. Ils sont donc souvent considérés comme phorétiques. Cependant, chez les larves de Parasitorhabditis proximi, Lazarevskaja (1962) a pu observer un léger développement et même la mue de nombreux individus dans le tube digestif d'Orthotomicus proximus. En outre, Ruhm et Chararas (1957) pour Parasitorhabditis hectographi chez Dryocoetes hectographus et Nickle (1963) pour Parasitorhabditis sp. chez Ips confusus ont signalé certains dommages causés par les larves de nématodes à l'épithélium intestinal de leur hôte. Ces observations amènent donc à considérer les Parasitorhabditis du tube digestif des Scolytides comme de véritables parasites. Toutefois, la possibilité qu'ils ont d'effectuer leur développement en l'absence d'hôte (Hunt et Poinar, 1971 ; Slobodyanyuc, 1974) conduit, comme l'a fait Poinar $(1972,1975)$ à les classer dans les parasites facultatifs. Il importe de préciser les caractères de $P$. ipsophila par rapport à cet ensemble d'observations.

L'action des Parasitorhabditis sur les populations de Scolytes n'a jamais été étudiée. Seules quelques observations ponctuelles font état d'une certaine mortalité. Ainsi, Ruhm et Chararas (1957) attribuent à $P$. hectographi la responsabilité de la mort de $D$. hectographus sous certaines conditions ; Fuchs (1915) chez Ips typographus et Blinova et coll. (1980) chez Ips subelongatus indiquent que l'obstruction du tube digestif par les nématodes peut aboutir à la mort de l'hôte dans certains cas d'infestations très lourdes.

Le cycle biologique d'I. sexdentatus a déjà été décrit par ailleurs (Lieutier, 1981); nous rappellerons simplement qu'il comporte en France deux générations annuelles et que les essaimages se situent fin avril début mai (génération d'hiver) et fin juillet début août (génération d'été). 


\section{1 - Méthodes et techniques}

Pendant tout le cycle de développement d'I. sexdentatus, des fragments d'écorce infestés, ainsi que la vermoulure se trouvant sur le tronc à l'endroit du prélèvement, sont récoltés sur des arbres attaqués en forêt de Fontainebleau et d'Orléans. Au laboratoire, les insectes sont disséqués afin d'observer le parasitisme par $P$. ipsophila. Les nématodes sont extraits de la vermoulure par une méthode dérivée de celle de Baermann, avec passage sur papier " kleenex ". Des élevages sont aussi réalisés au laboratoire, en contaminant des rondins de Pin sylvestre non infestés par des Scolytes porteurs de nématodes.

Pour la réalisation de montages in toto de tubes digestifs, les insectes sont disséqués dans la solution de Ringer, les tubes digestifs sont placés sur lame et fixés dans le Bouin alcoolique pendant 24 heures. Après élimination du fixateur, ils sont colorés par le glychémalum-érythrosine ou l'érytbrosine seule.

L'action sur les populations hôtes est étudiée sous trois aspects : mortalité, essaimage et ponte de l'hôte. L'étude de la mortalité est abordée par le biais de l'évolution des taux de parasitisme. Pour l'étude de l'essaimage, des écorces récoltées sur le terrain et abritant des $I p s$ en maturation sont rapportées au laboratoire; les insectes en essaimage sont obtenus selon la méthode décrite précédemment (Lieutier, 1981). Dans ce cas, le but de l'expérimentation étant de déterminer l'influence du parasitisme sur la précocité de sortie des insectes, il est indispensable que ceux-ci aient tous évolué dans les mêmes conditions. Il est donc impossible de prendre comme témoins des insectes totalement indemnes de parasites, obtenus au laboratoire. Comme la quasi totalité des $I p s$ sur le terrain sont porteurs de $P$. ipsophila au moment de leur essaimage (cf. infra), on est donc conduit à étudier l'influence du parasitisme sur l'essaimage par comparaison entre les insectes faiblement parasités et les insectes fortement parasités par ce Nématode. L'expérimentation porte sur deux lots : chacun d'eux est constitué d'écorces provenant d'un même arbre attaqué (Forêt de Fontainebleau) et récoltées dans les mêmes conditions d'exposition, de façon à ce qu'ils présentent chacun un maximum d'homogénéité. D'autre part, pour ne pas interférer avec l'action sur l'essaimage, du parasitisme par les Parasitaphelenchus ou $C$. diplogaster, il est nécessaire de porter son attention sur des insectes soit tous non parasités, soit tous parasités par ces deux derniers types de Nématodes, s'il n'y a pas de liaison entre le parasitisme du tube digestif et celui de la cavité générale ou du tissu adipeux (cf. infra). Le lot no 1 comprend 36 insectes uniquement parasités par $P$. ipsophila; le lot no 2 comprend 109 insectes tous parasités dans leur cavité générale ou leur tissu adipeux, en plus de $P$. ipsophila dans le tube digestif.

Pour l'étude de la ponte, des Scolytes femelles en ponte au laboratoire sont examinés à différents intervalles de temps après l'accouplement, selon la méthode déjà décrite (Lieutier, 1982). Les paramètres mesurés sont : la proportion de femelles ayant pondu au moment de l'observation, la longueur totale de la galerie maternelle, sa longueur depuis la chambre d'accouplement jusqu'à la première encoche de ponte, 
le nombre d'encoches de ponte, total et par centimètre de galerie (densité d'encoches), le nombre d'œufs déposés, total et par centimètre de galerie (densité de ponte) et la proportion d'encoches renfermant des œufs.

\section{2 - Résultats et interprétations des observations sur la biologie de P. ipsophila}

On observe les larves de $P$. ipsophila dans le tube digestif d'I. sexdentatus, principalement dans la partie terminale de l'intestin moyen et la partie proximale de l'intestin postérieur, jamais dans la cavité générale ou dans les tubes de Malpigbi.

L'étude des pourcentages d'infestation et de l'intensité des contaminations (nombre de Nématodes par insecte parasité) permet de suivre l'évolution du parasitisme au cours du développement de l'hôte (fig. 1 et 2).

\section{1 - Les contaminations}

Les Nématodes sont déjà présents dans le tube digestif de certaines larves et prénymphes d'I. sexdentatus mais leur nombre est peu élevé. Par contre, on n'en rencontre pas chez les nymphes, que ce soit dans le tube digestif en remaniement ou dans la cavité générale. Les quelques larves infestées semblent donc se décontaminer au moment de la nymphose; Slobodyanyuc (1974) indique cependant pour $P$. subelongati et $P$. piniperdae des possibilités de contamination au stade nymphal, comme au stade larvaire ou adulte de l'insecte hôte.

Chez les adultes, la contamination débute très tôt pour s'étendre rapidement et massivement, puisque de très nombreux insectes sont déjà contaminés peu de temps après la mue imaginale (stade blanc ou jaune). Ce phénomène est surtout net pour la génération d'hiver (68\% de contamination à ce stade) ; cette divergence entre génération s'explique sans doute par la différence de rapidité d'évolution des deux populations hôtes. La génération d'été atteint en effet le stade jaune au mois de juin, c'est-à-dire à une époque où les températures sont très favorables à une évolution rapide des insectes et donc de leur pigmentation; le stade jaune dure alors peu de temps, ce qui fait que les contaminations semblent plus tardives quand elles sont exprimées en fonction de la maturation de l'hôte.

L'augmentation rapide du nombre des contaminations au début de la maturation de l'insecte s'accompagne d'une augmentation également rapide de l'intensité du parasitisme, si bien que dès le stade brun clair ou brun, la quasi-totalité des insectes se trouve parasitée par un grand nombre de Nématodes ; ce parasitisme élevé dure jusqu'à l'essaimage. Pendant cette période, on peut observer parfois jusqu'à 85 larves de $P$. ipsophila dans le tube digestif d'I. sexdentatus.

Il n'a pas été possible de déterminer la voie d'entrée des larves de $P$. ipsophila dans le tube digestif d'I. sexdentatus mais Slobodyanyuc (1974) signale que les larves de $2^{e}$ stade de $P$. subelongati (larves infestantes) pénètrent dans le tube digestif d'I. subelongatus par l'anus; il est possible qu'il en soit de même pour $P$. ipsophila. 


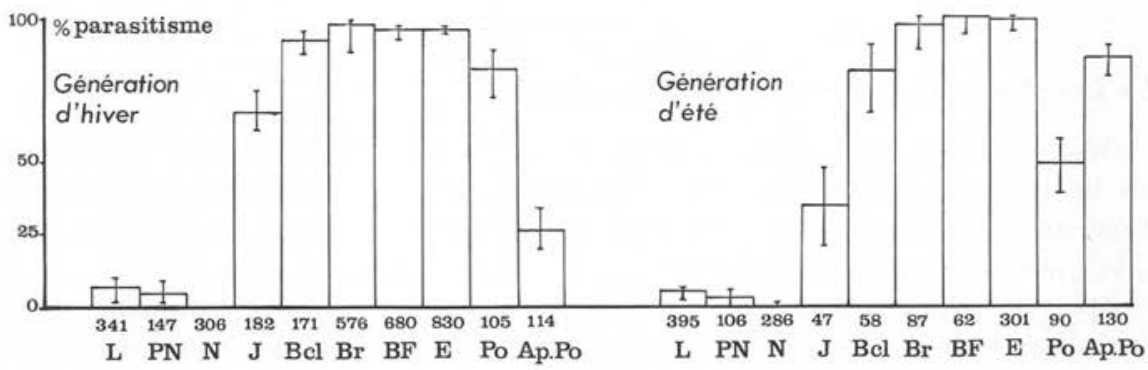

1

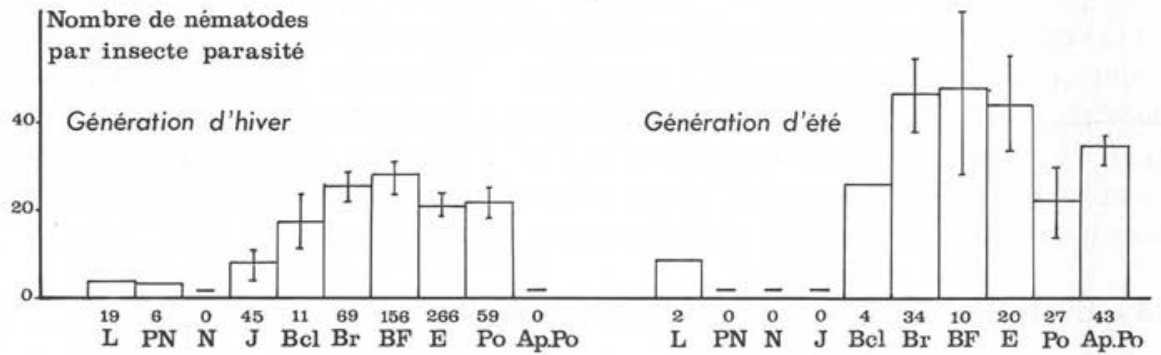

2

Proportion de nématodes dans le mésentéron (\%)

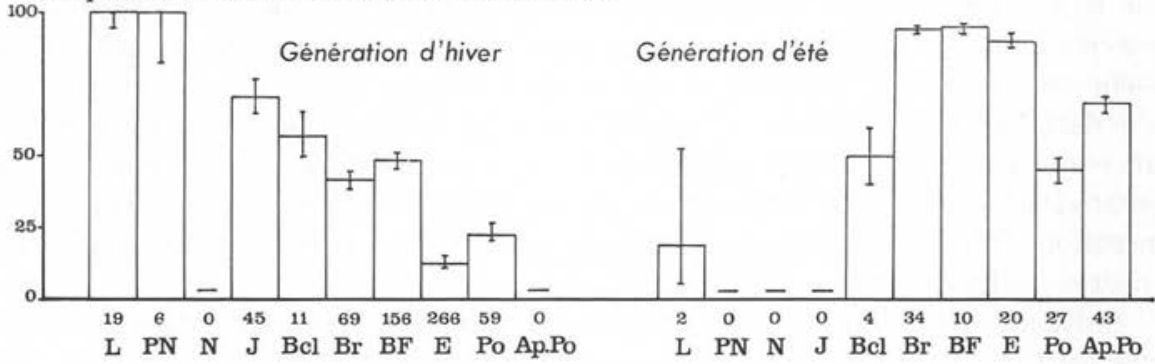

3

FIg. I. - Taux de parasitisme (pourcentage d'insectes parasités) d'Ips sexdentatus par P. ipsophila au cours des générations d'hiver et d'été. Les nombres d'insectes examinés sont indiqués sous les histogrammes. Les intervalles de confiance sont calculés pour un coefficient de sécurité de $95 \% . \mathrm{L}=$ larves; $\mathrm{PN}=$ prénymphes; $\mathrm{N}=$ nymphes; $\mathrm{J}=$ adultes en maturation, de couleur blanche ou jaune ; $\mathrm{B} . \mathrm{cl}=$ adultes en maturation, de couleur brun clair ; $\mathrm{Br}=$ adultes en maturation, de couleur brune $; \mathrm{BF}=$ adultes en maturation, de couleur brun foncé ou noire $; \mathrm{E}=$ adultes en essaimage $; \mathrm{Po}=$ adultes en ponte $; \mathrm{Ap}=$ adultes ayant terminé leur ponte.

FIG. 2. - Intensité du parasitisme (nombre de nématodes par insecte parasité) d'I. sexdentatus par $P$. ipsophila au cours des générations d'hiver et d'été. Les nombres d'insectes examinés sont indiqués sous les histogrammes. Les intervalles de confiance sont calculés pour un coefficient de sécurité de $95 \%$. La signification des abréviations est la même que dans la figure $\mathrm{I}$.

FIG. 3. - Proportion ( $\%$ ) de larves de $P$. ipsophila localisées dans le mésentéron d'I. sexdentatus au cours des générations d'hiver et d'été. Les nombres d'insectes examinés sont indiqués sous les histogrammes. Les intervalles de con fiance sont calculés pour un coefficient de sécurité de $95 \%$. La signification des abréviations est la même que dans la figure $\mathrm{r}$. 


\section{2 - Les décontaminations et recontaminations}

Après l'attaque d'un nouvel arbre, les insectes se décontaminent rapidement, ainsi que le montre la diminution très nette du pourcentage et de l'intensité du parasitisme, au moment de la ponte en juillet-août (génération d'été) ou à la fin de celle-ci en avril-mai (génération d'biver).

Après la ponte, au moins pour la génération d'été, des recontaminations des insectes semblent possibles, probablement par les descendants des Nématodes qui les infestaient auparavant, puisque le nombre d'insectes parasités et le nombre de Nématodes par insectes augmentent à nouveau. Ces recontaminations seraient permises par la rapidité d'évolution des populations de Nématodes et de Scolytes à cette époque ce qui explique qu'on ne les observe pas pour la génération d'hiver. Pour Parasitorhabditis piniperdae associé à Parasitaphelenchus papillatus chez B. destruens, Laumond et Carle (1971) ont également suggéré l'existence de recontaminations des insectes parents. Il est possible aussi qu'un certain nombre de Nématodes restent assez longtemps sur leur hôte avant de gagner les galeries.

\section{3 - Évolution des Nématodes dans l'insecte}

La figure 3 retrace les variations de la proportion de Nématodes localisés dans l'intestion moyen, par rapport au nombre total de ceux trouvés dans le tube digestif, au cours du développement d'I. sexdentatus. Le pourcentage de Nématodes localisés dans le mésentéron diminue assez régulièrement pendant toute la maturation de l'insecte, alors qu'au même moment le pourcentage et l'intensité du parasitisme restent assez élevés. $P$. ipsophila se localise donc d'abord dans l'intestin moyen, puis passe dans l'intestin postérieur. Cette migration est visible pour les deux générations mais elle est bien plus rapide pour celle d'été. L'augmentation de la proportion de Nématodes localisés dans l'intestin moyen au moment des recontaminations de la génération d'été (après la ponte) prouve également que la première localisation des parasites est bien le mésentéron.

Cette observation paraît difficilement conciliable avec une pénétration des Nématodes par l'anus, à moins de supposer, pour justifier une telle migration, que la localisation dans l'intestin moyen correspond à un besoin des Nématodes, pour atteindre un certain état de maturité par exemple. Cependant, la morphologie des larves de $P$. ipsophila de l'intestin moyen nous est apparue tout à fait semblable à celle des larves de l'intestin postérieur, de même que celle des larves présentes chez les insectes en début de maturation (coloration jaune à brun clair) est semblable à celle des larves qui contaminent les insectes en essaimage. Il ne semble donc pas y avoir d'évolution dans l'hôte, contrairement au cas de P. proximi et P. curvidentis (Lazarevskaja, 1962, 1965a, b) mais conformément aux observations de Fuchs (1937), Ruhm (1956) et Slobodyanyuc (1974) sur d'autres Parasitorhabditis du tube digestif.

\section{4 - Observations dans les galeries}

$P$. ipsophila est une espèce très fréquente; tous ses stades de développement s'observent dans les galeries pendant toute la période de présence des Scolytides, ce 
qui tend à prouver l'existence de générations libres chez cette espèce, comme chez de nombreux autres Parasitorhabditis (Lazarevskaja, 1961, 1962 ; Hunt et Poinar, 1971 ; Slobodyanyuc, 1974). Toutefois, une telle observation peut aussi correspondre à l'existence de migrations très échelonnées des Nématodes depuis les insectes parents vers les galeries. Après installation des $I p s$ sur rondins sains, nous avons noté l'apparition des premiers adultes de $P$. ipsophila dans les galeries de l'insecte au bout de quelques jours, ce qui correspond aux observations rapportées plus haut sur l'évolution du parasitisme. Cependant l'échelonnement de la migration des Nématodes vers les galeries, ainsi que l'existence possible de cycles libres dans celles-ci expliquent que l'importance des adultes de $P$. ipsophila dans ce milieu atteigne son maximum assez tard, au moment où les larves de Scolyte sont en fin de développement (Lieutier, 1979a). La sex-ratio de $P$. ipsophila dans les galeries est de 1,5 femelles pour 1 mâle

\section{5 - Relations de $\boldsymbol{P}$. ipsophila avec les Nématodes de la cavité générale ou du tissu adipeux}

L'étude porte sur les stades de l'insecte n'ayant pas subit de mortalité par parasitisme, c'est-à-dire jusqu'au stade adulte brun foncé (Lieutier, 1979b, 1980).

\subsection{1 - Relations entre P. ipsophila et les Parasitaphelenchus}

Pour chaque stade de développement des insectes, on note respectivement le nombre d'insectes non parasités, le nombre d'insectes parasités par l'une ou l'autre ou les deux sortes de Nématodes et les nombres théoriques attendus dans les mêmes catégories, compte tenu des pourcentages de parasitisme par chaque Nématode. La comparaison par test de $\mathrm{X}_{2}$ des valeurs observées et calculées, permet de juger s'il y a " cohabitation ", " exclusion " ou " indifférence " entre les deux parasites. Les résultats des tests ont montré quelque soit le stade de développement des insectes, que les différences entre valeurs observées et théoriques n'étaient jamais significatives au seuil de $95 \%$. Il y a donc indifférence entre les deux types de parasitisme, c'est-à-dire que la présence d'une espèce de nématode dans l'insecte n'influe pas sur la présence d'une autre espèce.

\subsection{2. - Relations entre P. ipsophila et Contortylenchus diplogaster}

Les calculs effectués de la même manière que précédemment, n'ont pas mis en évidence de relation entre ces deux espèces de parasites. Le parasitisme du tube digestif semble donc indépendant de celui de la cavité générale ou du tissu adipeux.

\section{3 - Actions de P. ipsophila sur les populations d'I. sexdentatus}

\section{1 - Action sur la mortalité, résultats et interprétations}

L'examen des figures 2 et 3 a montré que les variations du taux et de l'intensité du parasitisme par $P$. ipsophila s'expliquaient simplement par les modalités de la contamination et de la décontamination des insectes, liées au cycle du Nématode. 
A aucun moment une chute du parasitisme ne permet de conclure à une mortalité d'I. sexdentatus causée par P. ipsophila. Cependant, Ruhm et Chararas (1957) chez D. hectographus et Blinova et coll. (1980) chez I. subelongatus affirment que Parasitorhabditis peut causer la mort de l'insecte-hôte.

Il est possible que notre étude de l'évolution du taux et de l'intensité du parasitisme ne fasse pas apparaître une mortalité par les Nématodes, si celle-ci se situe au moment de la ponte des Insectes ou si elle ne survient pas à un stade précis de leur développement. Cependant, dans le cas de $D$. hectographus parasité par P. hectographi, les auteurs ont réalisé leurs observations par comparaison des dissections d'insectes morts à celles des insectes vivants, sur un petit nombre d'individus. Par ailleurs, dans le cas d'I. subelongatus parasité par $P$. subelongati, la mort de l'hôte, par obstruction intestinale, ne survient qu'en cas de contaminations très fortes (jusqu'à 200 Nématodes dans un même insecte). Cette intensité très élevée de parasitisme est cependant rare et l'obstruction intestinale ne représente donc qu'une cause fortuite de mortalité chez les Scolytides. En conséquence et compte tenu des résultats notés pour $P$. ipsophila, des recherches complémentaires paraissent nécessaires avant de conclure à une mortalité causée par les Parasitorhabditis chez les insectes qu'ils contaminent.

\section{2 - Action sur l'essaimage, résultats et interprétation ( $f i g .4$ )}

Les résultats sont semblables pour les deux lots et montrent que les insectes qui essaiment le plus tardivement sont ceux qui sont fortement parasités par $P$. ipsophila. Le retard semble donc d'autant plus prononcé que le parasitisme est plus intense, ce qui particulièrement net pour le lot no 2 .

Grâce au relevé des dates de sortie, il est possible de chiffrer approximativement ce retard. On peut, en effet, prendre comme point ce repère approximatif pour une catégorie donnée sa date de demi-essaimage correspondant à la sortie de $50 \%$ des insectes de cette catégorie. Ainsi, dans le lot no 1 , les insectes fortement parasités accusent un retard de deux jours sur les insectes faiblement parasités ; ce retard est d'une dizaine de jours dans le not no 2. La différence entre les deux lots s'explique sans doute par le fait que les catégories "faibles » et "fortes " n'ont pas la même signification dans les deux cas.

Deux hypothèses peuvent être avancées pour expliquer ces observations :

Première hypothèse : P. ipsophila retarde la maturation des insectes qu'il parasite.

Dans ce cas, étant donné leur localisation, l'action des Nématodes peut avoir lieu par détournement de substances nutritives ou par perturbation de leur assimilation par l'hôte. Or on a signalé précédemment qu'il ne semble pas y avoir d'évolution des larves de $P$. ipsophila dans le tube digestif de leur hôte ; leur alimentation y est sans doute très faible. Si elles retardent la maturation du Scolyte, c'est alors probablement par perturbation de l'assimilation. 


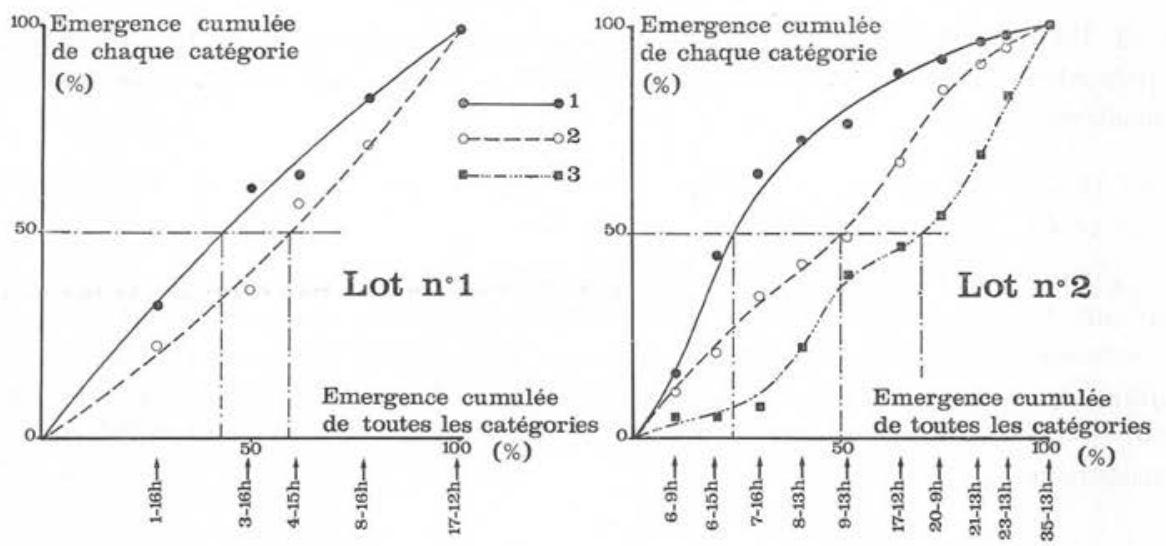

FIG. 4. - Émergence au laboratoire, des I. sexdentatus parasités par P. ipsophila. Les jours et heures indiqués sur l'échelle des abscisses correspondent aux dates des captures des insectes essaimants, le jour I étant celui où débute l'émergence.

Lot no $I$ : insectes parasités uniquement par $P$. ipsophila.

Courbe I : intensité de parasitisme inférieure à ${ }_{5} 5$ larves de $P$. ipsophila par insecte.

Courbe 2 : intensité de parasitisme supérieure ou égale à $1_{5}$ larves.

Lot no 2 : insectes tous parasités dans la cavité générale ou le tissu adipeux en plus de $P$. ipsophila dans le tube digestif.

Courbe I : intensité de parasitisme inférieure à Io larves de $P$. ipsophila par insecte.

Courbe 2 : intensité de parasitisme comprise entre ro et 20 larves de $P$. ipsophila par insecte.

Courbe 3 : intensité de parasitisme supérieure à 20 larves de $P$. ipsophila par insecte.

Deuxième hypothèse: les contaminations sont d'autant plus importantes que les insectes restent plus longtemps dans les galeries.

Cette explication est totalement opposée à la première puisqu'elle considère le parasitisme élevé comme une conséquence du retard à l'essaimage. Elle n'est cependant pas à rejeter puisque nous avons noté précédemment que les contaminations avaient lieu pendant tout le développement des insectes adultes. Néanmoins, le retard à l'essaimage ne porte que sur quelques jours et il paraît peu probable que ce délai suffise pour passer d'un faible à un fort parasitisme, alors qu'il a fallu plus d'un mois pour passer d'un parasitisme nul à un parasitisme faible. En outre, on devrait dans ce cas, noter une augmentation brutale et importante de l'intensité du parasitisme par $P$. ipsophila à la fin de la maturation de préessaimage, ce qui ne se trouve pas confirmé par la figure. En conséquence, la première hypothèse paraît la plus vraisemblable.

\section{3 - Action sur la ponte}

\subsection{1. - Résultats}

La nécessité de ne faire porter l'étude que sur les insectes dont la cavité générale et le tissu adipeux ne sont pas parasités, alliée à la lourdeur de l'expérimentation 
et de l'obtention des résultats, fait que certaines catégories sont très faiblement représentées. Celles dont l'effectif est inférieur à 4 individus ne sont pas prises en considération.

\subsubsection{1. - Pourcentage d'insectes ayant pondu au moment de l'observation (tableau I)}

Soixante pour cent des insectes faiblement parasités ont commencé à pondre au bout de $18 \mathrm{~h}$, contre $5 \%$ seulement des insectes fortement parasités et $14 \%$ des insectes au parasitisme moyen mais, dans ce dernier cas, la différence avec les insectes faiblement parasités n'est pas significative. En 24 heures, 70 à $80 \%$ et en 3 jours $100 \%$ des insectes ont commencé à pondre, quelle que soit l'intensité de leur parasitisme.

Tableau I. - Pourcentage d'insectes en ponte après différents délais suivant l'accouplement, pour différentes intensités de parasitisme. Dans chaque case, les valeurs portées sur la deuxième ligne correspondent aux limites de l'intervalle de confiance au niveau $95 \%$. La différence entre deux nombres est significative quand les cases qui les contiennent sont marquées de la même lettre. Le nombre d'insectes examinés est indiqué entre parenthèses.

\begin{tabular}{|c|c|c|c|c|c|}
\hline & $12 \mathrm{~h}$. & $18 \mathrm{~h}$. & $24 \mathrm{~h}$. & $3 \mathrm{j}$. & $6 \mathrm{j}$. \\
\hline $\begin{array}{l}\text { Parasitisme } \\
\text { Faible } \\
\text { (moins de } 10 \text { néma- } \\
\text { todes par insecte) }\end{array}$ & - & $\begin{array}{c}60 \% \\
14,8 \%-94,6 \% \\
\text { (5) a }\end{array}$ & $\begin{array}{c}75 \% \\
34,8 \%-96,8 \% \\
\text { (8) }\end{array}$ & $\begin{array}{c}100 \% \\
39,8 \%-100 \% \\
\text { (4) }\end{array}$ & - \\
\hline $\begin{array}{c}\text { Parasitisme } \\
\text { moyen } \\
\text { (10 à } 20 \text { nématodes } \\
\text { par insecte) }\end{array}$ & $\begin{array}{c}0 \% \\
0 \%-60 \% \\
(4)\end{array}$ & $\begin{array}{c}14,8 \% \\
2,0 \%-37,0 \% \\
(14)\end{array}$ & $\begin{array}{c}81 \% \\
58,2 \%-94,6 \% \\
(21)\end{array}$ & $\begin{array}{c}100 \% \\
54,1 \%-100 \% \\
\text { (6) }\end{array}$ & $\begin{array}{c}100 \% \\
54,1 \%-100 \% \\
\text { (6) }\end{array}$ \\
\hline $\begin{array}{l}\text { Parasitisme } \\
\text { fort } \\
\text { (plus de } 20 \text { nématodes } \\
\text { par insecte) }\end{array}$ & $\begin{array}{c}0 \% \\
0 \%-32,6 \% \\
\text { (9) }\end{array}$ & $\begin{array}{c}5 \% \\
0 \%-23,0 \% \\
(20) \quad a\end{array}$ & $\begin{array}{c}69,6 \% \\
47,4 \%-86,8 \% \\
(23)\end{array}$ & $\begin{array}{c}100 \% \\
47,8 \%-100 \% \\
\text { (5) }\end{array}$ & $\begin{array}{c}100 \% \\
59,0 \%-100 \% \\
\text { (7) }\end{array}$ \\
\hline
\end{tabular}

\subsubsection{2. - Longueur totale de la galerie maternelle}

Mesurée à différentes dates après l'accouplement $(3 \mathrm{~h}, 6 \mathrm{~h}, 12 \mathrm{~h}, 18 \mathrm{~h}, 24 \mathrm{~h}$, 3 jours, 6 jours), elle est une estimation de la vitesse de forage. Dans le cas d'un parasitisme faible (moins de 10 nématodes par insecte), la longueur totale de la galerie maternelle croît de $8 \pm 8,7 \mathrm{~mm}$ à la $3^{\mathrm{e}}$ heure après l'accouplement jusqu'à $94,5 \pm$ $15,8 \mathrm{~mm}$ au $3^{\mathrm{e}}$ jour. Dans le cas d'un parasitisme moyen (10 à 20 nématodes par insecte) elle croît de 10,5 $\pm 5,4 \mathrm{~mm}$ à la $3^{\mathrm{e}}$ heure jusqu'à $160 \pm 100,6 \mathrm{~mm}$ au $6^{\mathrm{e}}$ jour et, dans le cas d'un parasitisme fort (plus de 20 nématodes par insecte), de 7,7 \pm $3,5 \mathrm{~mm}$ à la $3^{\mathrm{e}}$ heure jusqu'à $118,1 \pm 43,5 \mathrm{~mm}$ au $6^{\mathrm{e}}$ jour. Cependant, les différences entre les 3 catégories de parasitisme ne sont jamais significatives au seuil de $95 \%$, 
quel que soit le temps écoulé depuis l'accouplement. P. ipsophila n'a donc pas d'action sur la vitesse de forage des galeries maternelles.

3.3.1.3. - Longueur de la galerie maternelle avant la première encoche de ponte.

Elle est de 14,8 $\pm 4,5 \mathrm{~mm}$ dans le cas d'un faible parasitisme (11 insectes), de $15,3 \pm 2,1 \mathrm{~mm}$ pour un parasitizme moyen ( 34 insectes) et de $15,5 \pm 2,3 \mathrm{~mm}$ pour un fort parasitisme (33 insectes). Les différences entre ces catégories ne sont pas significatives.

\subsubsection{4. - Longueur de la galerie de ponte}

Elle correspond à la distance entre le premier œuf et l'extrémité de la galerie maternelle. Mesurée à la $18^{\mathrm{e}}$ heure (début de ponte), à la $24^{\mathrm{e}}$ heure et au $3^{\mathrm{e}}$ jour après l'accouplement, elle ne dépend pas de l'intensité du parasitisme.

\subsubsection{5. - Nombre et densité des encoches et des œufs}

Seuls les insectes récoltés au bout de $24 \mathrm{~h}$ ou de 3 jours après l'accouplement sont en nombre suffisant pour donner lieu à des comparaisons valables (tableau II).

TABleau II. - Nombre et densité des encoches et des œufs dans les galeries d'I. sexdentatus examinées après différents délais suivant l'accouplement. Les valeurs des rapports encoches $/ \mathrm{cm}$, œufs/cm et œufs/encoches ont été obtenues à partir de celles calculées auparavant pour chaque insecte. La différence entre deux nombres est significative (niveau $95 \%$ ) quand la même lettre leur est affectée. Les nombres d'individus concernés sont indiqués entre parenthèses. Le parasitisme faible correspond à un nombre de nématodes par insecte inférieur à 10 ; le parasitisme moyen à un nombre compris entre 10 et 20 ; le parasitisme fort à un nombre supérieur à 20 . enc $=$ nombre total d'encoches; enc $/ \mathrm{cm}=$ nombre d'encoches par centimètre de galerie de ponte ; œufs $=$ nombre total d'œufs ; œufs $/ \mathrm{cm}=$ nombre d'œufs par centimètre de galerie de ponte $; œ u f s / e n c=$ taux d'encoches comportant des œufs.

\begin{tabular}{|c|c|c|c|c|c|c|}
\hline & \multicolumn{3}{|c|}{$\begin{array}{c}24 \mathrm{~h} . \\
\text { PARASITISME }\end{array}$} & \multicolumn{3}{|c|}{$\begin{array}{c}3 \mathrm{j} . \\
\text { PARASITISME }\end{array}$} \\
\hline & Faible & Moyen & Fort & Faible & Moyen & Fort \\
\hline Enc. & $2,83 \underset{(6)}{ \pm} 2,45$ & $2,7 \underset{(20)}{ \pm 0,66}$ & $\begin{array}{c}2,67 \pm 0,72 \\
(18)\end{array}$ & $18,6 \pm 11,3$ & $14,0_{(6)}^{ \pm} \underset{7,3}{ }$ & $13,4 \frac{ \pm 8,9}{(5)}$ \\
\hline $\mathrm{Enc} / \mathrm{cm}$ & $3,55 \underset{(6)}{ \pm} 1,07$ & $\begin{array}{c}1,78 \pm 0,43 \\
(20)\end{array}$ & $\begin{array}{c}3,21 \pm 0,60 \\
(17)\end{array}$ & - & $\begin{array}{c}2,82 \pm 0,5 \\
(6) \mathrm{a}\end{array}$ & $2,18 \pm 0,67$ \\
\hline Eufs & $1,80 \pm \frac{ \pm}{(5)} 1,52$ & $\begin{array}{c}1,78 \pm 1,11 \\
(18)\end{array}$ & $\begin{array}{c}1,61 \pm 0,59 \\
(18)\end{array}$ & $10,0 \pm \frac{ \pm}{(4)}$ & $6,17 \pm \frac{2,23}{(6)}$ & $7,0 \underset{(4)}{ \pm} 5,8$ \\
\hline Eufs/cm & $2,58 \underset{(5)}{ \pm} 1,48$ & $\begin{array}{c}2,21 \pm 0,61 \\
(18)\end{array}$ & $\begin{array}{c}2,08 \pm 0,67 \\
(17)\end{array}$ & - & $1,39 \underset{(6)}{ \pm} 0,71$ & $1,13 \pm 0,0,76$ \\
\hline Eufs/enc & $0,72 \pm 0,36$ & $\begin{array}{c}0,62 \pm 0,17 \\
(18)\end{array}$ & $\begin{array}{c}0,61 \pm 0,17 \\
(18)\end{array}$ & $0,58 \pm 0,13$ & $0,50 \pm \frac{ \pm}{(6)} 0,28$ & $0,56 \pm 0,0,54$ \\
\hline
\end{tabular}


Le troisième jour, la densité des encoches de ponte est significativement plus faible chez les insectes fortement parasités que chez les insectes moyennement parasités. On observe la même tendance à la $24^{\mathrm{e}}$ heure mais la différence n'est pas significative. Les autres paramètres semblent également plus faibles chez les insectes fortement parasités que chez ceux qui portent peu de parasites mais les différences ne sont pas non plus significatives.

\subsection{2. - Interprétation et discussion}

P. ipsophila a une action certaine sur la précocité de la ponte (tableau I) et la densité des encoches (tableau II). En outre, le fait que les valeurs de tous les paramètres du tableau II soient plus faibles lorsque le parasitisme est intense laisse supposer que celui-ci a une action sur ces paramètres, en particulier sur le nombre et la densité des œufs, bien que les différences ne soient pas significatives. $P$. ipsophila influencerait donc la ponte d'I. sexdentatus sous deux aspects : un démarrage plus tardif et un taux plus faible.

Cette influence est cependant très réduite puisque le retard est au maximum de 6 beures à la température du laboratoire (tableau I) et les variations de densité des encoches et des œufs relativement minimes (tableau II). De plus, aucune influence n'est visible quant aux caractéristiques de la galerie maternelle, pas même au niveau de sa longueur depuis la chambre d'accouplement jusqu'à la première encoche. Le retard dans le démarrage de la ponte n'est donc pas décelable au niveau de la quantité d'aliments nécessaire à la maturation du premier œuf, ce qui corrobore l'idée que le parasitisme par $P$. ipsophila n'a qu'une influence minime sur la reproduction de son hôte.

\section{4- Conclusions}

L'action de $P$. ipsophila sur les populations d'I. sexdentatus a été étudiée sur des insectes tous parasités, chez lesquels seule varie l'intensité du parasitisme. On a donc supposé implicitement que les modifications dues à celui-ci sont très progressives, pratiquement nulles dans le cas d'un parasitisme très faible et d'autant plus profondes que le nombre de parasites augmente. De fait, les résultats montrent la plupart du temps que les valeurs obtenues pour les différents paramètres, lors d'un parasitisme moyen, sont intermédiaires entre celles observées lors d'un parasitisme faible et celles observées lors d'un parasitisme fort. Il est donc permis de considérer comme peu vraisemblable qu'il existe une brusque discontinuité entre l'état non parasité et l'état parasité. Les différences observées entre insectes faiblement et fortement parasités doivent aussi exister entre insectes non parasités et insectes parasités.

On peut donc conclure que $P$. ipsophila cause un retard de quelques jours dans l'essaimage et de quelques heures dans l'initiation de la ponte d'I. sexdentatus. Par contre, il ne semble pas causer de mortalité et son action sur la reproduction paraît très faible. Toutefois, même faible, il existe incontestablement une action du parasite 
sur son hôte et il importerait de déterminer quelles en sont les modalités. Êtant donné la localisation des Nématodes, il est très probable que leur action s'exerce par spoliation en aliments ou par perturbation de l'assimilation. L'absence de développement apparent des larves de $P$. ipsophila dans le tube digestif de leur hôte suggère que leurs prélèvements sur le bol alimentaire et donc leur action spoliatrice doivent être limités. La perturbation de l'assimilation peut correspondre à une destruction de l'épithélium intestinal, par des procédés mécaniques ou chimiques ; elle peut correspondre aussi, sous l'influence de diverses substances que produiraient les Nématodes, à une modification du fonctionnement enzymatique de cet épithélium, de façon directe ou indirecte (action sur le système neuroendocrinien par exemple). L'étude histologique du tube digestif des insectes parasités devrait apporter des éléments de réponse.

Par ses caractères biologiques et son action sur Ips sexdentatus, P. ipsophila semble bien posséder les traits d'un parasite facultatif, mais l'absence de développement dans l'hôte et son action limitée sur les populations de ce dernier suggèrent que ce cas de parasitisme est encore très proche de la phorésie.

Remerciements : L'auteur remercie Monsieur C. Chararas, Directeur de Recherches au CNRS, membre de l'Institut, d'avoir bien voulu relire et critiquer le manuscrit. Il 1emercie également Messieurs Bonnaire et Tendron, chefs de Centre, Corbaz, Grillo, Lerouet, Trochesec, Vayssette et Vincq, chefs de secteurs, de l'Office National des Forêts, ainsi que Monsieur Rousseau, technicien au Service Régional d'Aménagement Forestier de la région Centre, pour l'aide qu'ils lui ont apporté lors de ses études sur le terrain et pouı la fourniture du matériel nécessaire aux élevages.

\section{BIBLIOGRAPHIE}

Blivona S. L., Korenchenko E. A., Ivanova E. C. : (En russe.) Essai d'élevage de scolyte dépourvus d'Helminthes et contamination expérimentale d'Ips subelongatus par différente espèces de nématodes. Les Helminthes des insectes, I980, Moscou, I8-21.

Fuchs A. G. : Die Naturgeschichte der Nematoden und einiger anderer parasiten r. des $I p s$ typographus - 2. des Hybolius abietis. Zool. Jahrb. (Syst), 1915, 38, 109-222.

Fuchs A. G. : Neue parasitische und halbparasitische Nematoden bei Borkenkäfern und einige andere Nematoden. Zool. Jahrb. (Syst), I937, 70, 29I-380.

Hunt R. S., Pornar G. O. : Culture of Parasitorhabditis sp. (Rhabditidae, Protorhabditinae) on a fungus. Nematologica, 1971, $17,321-322$.

LAUMOND C., CARLE P. : Nematodes associés et parasites de Blastophagus destruens Woll (Coleoptera : Scolytidae). Entomophaga, 1971, 16, 61-66.

Lazarevskaja S. L. : (En russe.) Sur la faune des nématodes de Acanthocinus aedilis (Cerambycidae). Helminthologia, 1961, 3, 21 I-220.

Lazarevskaja S. L. : (En russe.) Nématodes d'Orthotomicus laricis et Orthotomicus proximus de la province d'Orenburg (URSS). Helminthologia, 1962, 4, 254-265.

Lazareyskaja S. L. : (En russe.) Les nématodes des insectes déprédateurs des forêts : I. Caractéristique biologique des nématodes du genre Parasitorhabditis (Rhabditidae : Parasitorhabditinae). Trudy gel' mintol. lab. Akad. Nauk CCCP, 1965a, Is, 93-10o.

Lazarevskaja S. L. : (En russe.) Les nématodes des Coléoptéres du Pin (faune, biologie, systématique). Thèse, Moscou, r $1965 \mathrm{~b}, 20 \mathrm{p}$.

Lieutier F. : Les fluctuations des populations de nématodes associés aux Scolytides. Bull. Soc. Zool. Fr., I979a, 104, 423-433.

Lieutier F. : Le parasitisme d'Ips sexdentatus (Coleoptera : Scolytidae) par Contortylenchus diplogaster (Nematoda : Allantonematidae). Rev. Nematol., 1979b, 2, I43-I 5I.

Lieutier F. : Le parasitisme d'I ps sexdentatus (Boern) (Coleoptera : Scolytidae) par les nématodes du genre Parasitaphelenchus Fuchs. Relation avec le parasitisme par Contortylenchus diplogaster (V. LINS.). Rev. Nematol., 1980, 3, 271-281. 
Lieutier F. : Influence des nématodes parasites sur l'essaimage du Scolytide Ips sexdentatus (Boern). Action régulatrice du froid. Acta Oecologica. Oecol. applic., 1981, 2, 357-368.

Lieutier F. : Action des nématodes endoparasites sur la ponte du Scolytide Ips sexdentatus Boerner (Insecta : Coleoptera). Acta Oecologica. Oecol. applic., 1982, 3, I9I-204.

Lieutier F., LAumond C. : Nématodes parasites et associés à Ips sexdentatus et Ips typographus (Coleoptera : Scolytidae) en région parisienne. Nematologica, 1978, 24, 187-200.

Nickle W. R. : Observation on the effects of Nematodes on I ps confusus (Leconte) and other bark beetles. J. Insect. Pathol., 1963, s, 386-389.

Poinar G. O. : Nematodes as facultative parasites of insects. A Rev. Entomol., 1972, 17, 103-122.

Poinar G. O. : Entomogenous nematodes. A manual and host list of insect nematode associations. E. J. Brill, Leiden, 1975, 317 p.

Ruнm W. : Die Nematoden der Ipiden. Parasitol. SchrReihe, I956, 6, I-437.

RuHM W., Chararas C. : I. Einige neue mit Dryocoetes hectographus vergeselschaftete Nematodenarten. 2. Biologie et histologie de Dryocoetes hectographus et influence des nématodes du tube digestif. Entomophaga, 1957, 2, 253-269.

Slobodyanyuk O. V. : (En russe.) Particularités biologiques des nématodes du genre Parasitorhabditis (Rhabditidae). Trudy gel'mintol. Lab. Akad. Nauk CCCP, 1974, 24, 160-168. 